\title{
SHORT COVERING CODES ARISING FROM MATCHINGS IN WEIGHTED GRAPHS
}

\author{
ANDERSON N. MARTINHÃO AND EMERSON L. MONTE CARMELO \\ This work is dedicated to Professor Adilson Gonçalves
}

\begin{abstract}
The concept of embedded matching in a weighted graph is introduced, and the maximum cardinality of an embedded matching is computed. On the other hand, consider the following problem induced by a short covering. Given a prime power $q$, the number $c(q)$ denotes the minimum cardinality of a subset $\mathcal{H}$ of $\mathbb{F}_{q}^{3}$ which satisfies the following property: every element in this space differs in at most 1 coordinate from a scalar multiple of a vector in $\mathcal{H}$. As another goal, a connection between embedded matching and short covering code is established. Moreover, this link is applied to improve the upper bound on $c(q)$ for every odd prime power $q$.
\end{abstract}

\section{INTRODUCTION}

In the three-dimensional space $\mathbb{F}_{q}^{3}$ over the finite field $\mathbb{F}_{q}$, the set of all scalar multiples of vectors in $S$ is denoted by $\mathbb{F}_{q} \cdot S=\left\{\beta s: \beta \in \mathbb{F}_{q}\right.$ and $\left.s \in S\right\}$. As usual, the symbol $e_{j}$ represents the $j$-th canonical vector in $\mathbb{F}_{q}^{3}$, where $1 \leq j \leq 3$. A subset $\mathcal{C}$ of $\mathbb{F}_{q}^{3}$ is a covering of $\mathbb{F}_{q}^{3}$ when the sum set below is all the space, that is,

$$
\mathcal{C}+\mathbb{F}_{q} \cdot\left\{e_{1}, e_{2}, e_{3}\right\}:=\left\{c+s: c \in C \text { and } s \in \mathbb{F}_{q} \cdot\left\{e_{1}, e_{2}, e_{3}\right\}\right\}=\mathbb{F}_{q}^{3} .
$$

Kalbfleish and Stanton [7] proved that the minimum cardinality of a covering of $\mathbb{F}_{q}^{3}$ is $K_{q}(3,1)=\left\lceil q^{2} / 2\right\rceil$.

A generalization for higher dimensions $n \geq 3$ has been a great challenge of combinatorial coding theory since 1948. Carnielli 2 extended these numbers to arbitrary radius $R$. See an overview on covering codes in 3]. Updated tables on $K_{q}(n, R)$ are available in Kéri [8].

Covering code can be reformulated in terms of dominating set on graph, which is strongly $N P$-hard, according to [5]. Several variants have been introduced in order to shed light on the computation of the numbers $K_{q}(n, R)$, see the contributions 4. 15. on similar problems with algebraic constraints. In this work, we turn our attention to a recent variant described as follows. The subset $\mathcal{H}$ is a short covering of $\mathbb{F}_{q}^{3}$ if

$$
\mathbb{F}_{q} \cdot \mathcal{H}+\mathbb{F}_{q} \cdot\left\{e_{1}, e_{2}, e_{3}\right\}=\mathbb{F}_{q}^{3}
$$

Received by the editor April 12, 2011 and, in revised form, August 24, 2011.

2010 Mathematics Subject Classification. Primary 11B75, 05C70, 94B75; Secondary 05B40, 11T71, 94B25.

Key words and phrases. Matching, weighted graph, square number, finite field, independent vectors, covering codes.

The first author was supported by Capes.

The second author is supported by Fundação Araucária and CNPq. 
Thus both kinds of coverings are strongly connected because $\mathcal{H}$ is a short covering if and only if the set $\mathbb{F}_{q} \cdot \mathcal{H}$ generates a covering of $\mathbb{F}_{q}^{3}$. Note that a short covering $\mathcal{H}$ is invariant under scalar multiplication. The induced extremal problem $c(q)$ is defined as the minimum cardinality of a short covering $\mathcal{H}$.

This problem can be also extended to arbitrary dimension. The study of short covering codes is motivated by computational and theoretical reasons. Indeed, short coverings might provide us with a way to store nonlinear codes using less memory than the classical ones. Their results might reach record-breaking on the classical codes, as obtained in [11. On the theoretical viewpoint, results on short covering have been investigated in connection with additive combinatorics, graph theory, and group theory. In particular, the contribution [13 presents a construction of short covering codes from sum-free sets (see [14 also). By using this tool, the following upper bound is obtained in 12 . Let $q$ be a prime power.

- If $q$ is odd, then $c(q) \leq 6\left\lceil\frac{q-1}{12}\right\rceil+6\left\lceil\log _{4}\left(\frac{q-1}{4}\right)\right\rceil+3$.

- If $q$ is even, then $c(q) \leq 6\left\lceil\frac{q-1}{9}\right\rceil+6\left\lceil\log _{4}\left(\frac{q-1}{3}\right)\right\rceil+3$.

In this work, we improve the above upper bound when $q$ is odd, more precisely.

Theorem 1.1. Given $q$ an odd prime power:

(1) If $q \equiv 1(\bmod 4)$, then $c(q) \leq(q+5) / 2$.

(2) If $q \equiv 3(\bmod 4)$, then $c(q) \leq(q+3) / 2$.

A comparative analysis with the lower bound $\lceil(q+1) / 2\rceil \leq c(q)$ (see [13]) reveals that Theorem 1.1 is very close to the optimal value: the gap is at most 2 . Moreover, Theorem 1.1 yields sharp bound at least for $q=7$; according to 10 .

The set of all square numbers is a subgroup of index 2 of the multiplicative group $\mathbb{F}_{q}^{*}$, if $q$ is an odd prime power. This fact is used in the proof of Theorem 1.1 (see Theorem 4.2). Since this fact does not hold for the case where $q$ is even, our method seems to be unable to reach this case. Any improvement on the numbers $c\left(2^{r}\right)$ would be desirable.

A little unexpectedly, the constructions of these sharper short coverings are based on embedded matchings, the main tool. The work is structured as follows. Upper bounds and constructions of optimal embedded matchings are established in Sections 2 and 3. On the other hand, the connection between embedded matching and the short covering code is described in Section 4. Finally, Theorem 1.1 is proved in Section 5 ,

\section{The Main tool: Embedded MATChing}

Let $G=(V, E)$ denote a simple graph composed by the vertex set $V$ and the edge set $E$. As usual, a vertex $x$ is incident with the edge $e$ if $x \in e$. The subset $M$ of the edges is a matching of $G$ if $e \cap f=\emptyset$ for any two distinct edges $e, f \in M$. The matching $M$ matches $x$ if $x$ is incident with an edge in $M$. Applications, computational and mathematical results on matchings are collected in Lovász and Plummer [9].

Several concepts of weights in graphs have been investigated: vertex-weighted, weighted graph matching, etc. The search for maximal matching in these environments deals mainly with optimization problems (maximum sum of the weights in a matching, for instance, see [1]).

For our purposes, a weighted graph $(G, \omega)$ is a simple graph $G=(V, E)$ such that each edge $e$ is assigned to its weight $\omega(e)$. As usual, $K_{n}$ denote the complete graph 
whose vertex set is $V=[n]=\{1,2, \ldots, n\}$. The edge $\{x, y\}$ will be represented by the pair $(x, y)$, under the convention that $x>y$. From now on, we focus on the weighted graph $\left(K_{n}, \omega\right)$, where

$$
\begin{aligned}
\omega: E\left(K_{n}\right) & \longrightarrow[n-1], \\
(x, y) & \longmapsto x-y .
\end{aligned}
$$

We introduce the following kind of matching.

Definition 2.1. Consider the weighted graph $\left(K_{n}, \omega\right)$. The set $M$ is called an embedded matching of $\left(K_{n}, \omega\right)$ if $M$ satisfies the following properties:

- the edge set $M$ is a matching of the simple graph $K_{n}$,

- (injective condition): the edges in $M$ have distinct weights, that is, $\omega(e) \neq$ $\omega(f)$ for every $e \neq f$ in $M$,

- the image $\omega(M)$ is contained in $[\lfloor n / 2\rfloor]=\{1,2, \ldots,\lfloor n / 2\rfloor\}$.

The weighted graph $\left(K_{n}, \omega\right)$ can be associated to the bipartite graph $\Gamma_{n}=$ $(\bar{V}, \bar{E})$, where $\bar{V}=E\left(K_{n}\right) \cup[n-1]$ and $\bar{E}=\left\{\{e, \omega(e)\}: e \in E\left(K_{n}\right)\right\}$. An edge subset $M$ in $\left(K_{n}, \omega\right)$ induces naturally the edge subset $\bar{M}=\{\{f, \omega(f)\}: f \in M\}$ of $\Gamma_{n}$. It is worth mentioning that the injective condition in Definition 2.1 corresponds to the fact that $\bar{M}$ is a matching in $\Gamma_{n}$.

Perfect embedded matchings happen less often than perfect matchings in $K_{n}$. For instance, there are 3 perfect matchings in $K_{4}$, namely,

$$
\{(4,3),(2,1)\}, \quad\{(4,2),(3,1)\}, \quad\{(4,1),(3,2)\},
$$

but none of them satisfies Definition 2.1. Since the additional conditions imposed in Definition 2.1 are restrictive, it seems to be interesting to analyze the behavior of an optimal embedded matching.

Definition 2.2. For every $n \geq 2$, define

$$
\Omega(n)=\max \left\{|M|: M \text { is an embedded matching of }\left(K_{n}, \omega\right)\right\} .
$$

Since an embedded matching of $\left(K_{n}, \omega\right)$ is always a matching in $K_{n}$, the upper bound $\Omega(n) \leq n / 2$ is derived trivially. This bound may be slightly improved under certain conditions.

Proposition 2.3. For any integer $n \geq 2$, the upper bound holds:

$$
\Omega(n) \leq \begin{cases}\lfloor n / 2\rfloor-1, & \text { if } n \equiv 4 \text { or } 6(\bmod 8), \\ \lfloor n / 2\rfloor, & \text { otherwise. }\end{cases}
$$

Proof. Choose an $n$ such that $n \equiv 4$ or $6(\bmod 8)$. Suppose for a contradiction that there is an embedded matching $M$ of $\left(K_{n}, \omega\right)$ with $|M|=n / 2$, thus $M$ is perfect. Consider the following partition of $M$ :

$$
\begin{aligned}
& X=\{(a, b) \in M: a \text { and } b \text { have distinct parities }\} \\
& Y=\{(a, b) \in M: a \text { and } b \text { are even }\} \\
& Z=\{(a, b) \in M: a \text { and } b \text { are odd }\}
\end{aligned}
$$

and let $x=|X|, y=|Y|$, and $z=|Z|$. There are $n / 2$ even vertices and $n / 2$ odd vertices matched by $M$. Each even vertex is matched by either an edges in $X$ or 
one edge in $Y$, and each odd vertex is only matched by edges in $X$ or $Z$. The diophantine equations

$$
\left\{\begin{array}{l}
x+2 y+0 z=n / 2 \\
x+0 y+2 z=n / 2
\end{array}\right.
$$

hold from the fact that $M$ is perfect. On the other hand, let us now analyze the impact of the weights. Edges with odd weight are generated by the edges in $X$, while edges with even weight come from $Y$ or $Z$. The embedding $\omega$ into $[\lfloor n / 2\rfloor]$ implies the equalities

$$
x=\lceil n / 4\rceil \text { and } y+z=\lfloor n / 4\rfloor .
$$

The argument is divided into two cases:

Case 1 . When $n \equiv 4(\bmod 8)$, the equalities $x=y+z=n / 4$ hold by (2.2) $)$ By combining with (2.1), we obtain $2 y=n / 4$, contradicting the fact that $n / 4$ is odd.

Case 2. If $n \equiv 6(\bmod 8)$, a simple counting yields $x=(n+2) / 4$ and $y+z=$ $(n-2) / 4$. Again by (2.2), $x=(n+2) / 4$ and $y+z=(n-2) / 4$ hold. The system in (2.1) implies $2 y=(n-2) / 4$, a contradiction.

Because the upper bound $\Omega(n) \leq n / 2$ holds for any $n \geq 2$, the proof is complete.

\section{Constructions of matchings in $\left(K_{n}, \omega\right)$}

In this section, we focus on constructions of maximal matchings. A simple but very useful tool is stated below.

Definition 3.1. Let $M$ and $N$ be embedded matchings of $\left(K_{n}, \omega\right)$. We say that $M$ and $N$ are totally disjoint when:

- the sets formed by the vertices matched by $M$ and $N$ are disjoint,

- the images $\omega(M)$ and $\omega(N)$ are disjoint sets also.

Lemma 3.2. Let $M$ and $N$ be embedded matchings of $\left(K_{n}, \omega\right)$. Suppose that $M$ and $N$ are totally disjoint. Then the union $M \cup N$ is also an embedded matching of $\left(K_{n}, \omega\right)$.

Proof. The proof is immediate.

The first goal of this work concerns with the exact values of such matchings.

Theorem 3.3. Let $n \geq 2$ be a positive integer.

(1) If $n$ is odd, then $\Omega(n)=\lfloor n / 2\rfloor$.

(2) For an even $n, \Omega(n)= \begin{cases}\lfloor n / 2\rfloor, & \text { if } n \equiv 0 \text { or } 2(\bmod 8), \\ \lfloor n / 2\rfloor-1, & \text { if } n \equiv 4 \text { or } 6(\bmod 8) \text {. }\end{cases}$

Before proceeding the proof of Theorem 3.3. let us mention briefly the approach used. In order to construct an embedded matching $M$, the main heuristic is based on the steps below:

Step 1: Choose a suitable edge $(a, b)$ in $M$, with $a>b$.

Step 2: Choose the next pair from the previous one according to the rule: decrease 1 in the first coordinate and increase 1 in the second coordinate.

Step 3: Repeat step 2 until the $(a, b)$ reaches $a, b$, or $a-b$ as a "forbidden" number. 
Since all the edges generated have the same parity, the second embedded matching $N$ is obtained by running the heuristic again. Under certain conditions, we can apply Lemma 3.2. Since the union $M \cup N$ is not necessarily maximal, this recursive procedure is repeated as many times as possible.

Proof of Theorem 3.3. We divide the proof into several cases.

Case 1 . The begin with the case where $n$ is odd.

Case 1.1. If $n=4 t-1$, then choose

$$
\begin{aligned}
& M=\{(2 t, 1),(2 t-1,2), \ldots,(t+1, t)\}, \\
& N=\{(4 t-1,2 t+1),(4 t-2,2 t+2), \ldots,(3 t+1,3 t-1)\},
\end{aligned}
$$

which are totally disjoint matchings of $\left(K_{n}, \omega\right)$. Indeed, it is easy to see that both $M$ and $N$ are disjoint matchings of the complete graph $K_{n}$, because $M$ and $N$ match disjoint vertex sets. Moreover, their weights induce the partition of $[\lfloor n / 2\rfloor]=$ $[2 t-1]$ into odd and even numbers, namely, $\omega(M)=\{1,3, \ldots, 2 t-1\}$, and $\omega(N)=$ $\{2,4, \ldots, 2 t-2\}$. Since both $M$ and $N$ are embedded matchings of $\left(K_{n}, \omega\right)$, the application of Lemma 3.2 implies that $M \cup N$ is also an embedded matching of $\left(K_{n}, \omega\right)$.

Case 1.2. If $n=4 t-3$, we can choose the following sets:

$$
\begin{aligned}
& \{(2 t-2,1),(2 t-3,2), \ldots,(t, t-1)\}, \\
& \{(4 t-3,2 t-1),(4 t-4,2 t), \ldots,(3 t-1,3 t-3)\} .
\end{aligned}
$$

Analogously to the previous case, these sets produce totally disjoint embedded matchings of $\left(K_{n}, \omega\right)$, whose union is an embedded matching of $\left(K_{n}, \omega\right)$, by Lemma 3.2 .

In both cases we built matchings of $\left(K_{n}, \omega\right)$ that reach the sharp upper bound in Proposition 2.3 .

Case 2. Now choose an even positive integer $n$. By Proposition 2.3 , there is no hope of building a perfect embedded matching of $\left(K_{n}, \omega\right)$ for the cases where $n \equiv$ 4 or $6(\bmod 8)$.

Case 2.1. Let $n=8 t+4$ for some integer $t \geq 1$. The sets

$$
\begin{aligned}
& \{(4 t+2,1),(4 t+1,2), \ldots,(2 t+2,2 t+1)\}, \\
& \{(8 t+3,4 t+3),(8 t+2,4 t+4), \ldots,(6 t+4,6 t+2)\}
\end{aligned}
$$

are totally disjoint matchings of $\left(K_{n}, \omega\right)$. We apply Lemma 3.2 on these matchings, then their union produces an embedded matching of $\left(K_{n}, \omega\right)$. The cardinalities of these matchings reveal that the upper bound in Proposition 2.3 is sharp.

Case 2.2. If $n=8 t+6$ for some $t \geq 1$. Note that the sets

$$
\begin{aligned}
& \{(4 t+4,1),(4 t+3,2), \ldots,(2 t+3,2 t+2)\}, \\
& \{(8 t+5,4 t+5),(8 t+4,4 t+6), \ldots,(6 t+6,6 t+4)\}
\end{aligned}
$$

are totally disjoint matchings of $\left(K_{n}, \omega\right)$. Again, by applying Lemma 3.2, the union induces an embedded matching of $\left(K_{n}, \omega\right)$ with maximal cardinality, by Proposition 2.3 .

The remaining cases need more elaborate constructions, as described below. 
Case 2.3. If $n \equiv 0(\bmod 8)$, then $\Omega(n)=\lfloor n / 2\rfloor$. Particular embedded matchings of $\left(K_{8}, \omega\right)$ and $\left(K_{16}, \omega\right)$ are, respectively,

$$
\begin{aligned}
& \{(2,1),(7,5),(6,3),(8,4)\}, \\
& \{(3,2),(13,11),(7,4),(14,10),(6,1),(15,9),(12,5),(16,8)\} .
\end{aligned}
$$

Suppose that $n=8 t$ for some $t \geq 3$. Consider the sets

$$
\begin{aligned}
& M_{1}=\{(8 t, 4 t),(8 t-1,4 t+1), \ldots,(6 t+1,6 t-1)\}, \\
& M_{2}=\{(6 t, 2 t+1),(2 t+2,1),(t+1, t)\}, \\
& M_{3}=\{(2 t+3,2 t),(2 t+4,2 t-1), \ldots,(3 t+1, t+2)\}, \\
& M_{4}=\{(3 t+2, t-1),(3 t+3, t-2), \ldots,(4 t-1,2)\} .
\end{aligned}
$$

Note that $M_{1}, M_{2}, M_{3}$, and $M_{4}$ are matchings of the simple graph $K_{n}$. Moreover, their edges have distinct weights. Because $M_{1}, M_{2}, M_{3}$, and $M_{4}$ are pairwise totally disjoint, an application of Lemma 3.2 implies that $M=\bigcup_{i=1}^{4} M_{i}$ is an embedded matching of $\left(K_{n}, \omega\right)$. Since $|M|=4 t$, Proposition 2.3 yields that $M$ reaches the maximum cardinality.

It is worth mentioning that the construction for $t \geq 3$ does not hold for $t=1$ or $t=2$, because the sets $M_{1}, M_{2}, M_{3}$ and $M_{4}$ would not be totally disjoint for these situations.

Case 2.4. If $n \equiv 2(\bmod 8)$, then $\Omega(n)=\lfloor n / 2\rfloor$. Particular matchings of $\left(K_{10}, \omega\right)$ and $\left(K_{18}, \omega\right)$ are, respectively,

$$
\begin{aligned}
& \{(2,1),(9,7),(6,3),(8,4),(10,5)\}, \\
& \{(7,6),(15,13),(4,1),(16,12),(8,3),(17,11),(9,2),(18,10),(14,5)\} .
\end{aligned}
$$

Assume now $n=8 t+2$ for some $t \geq 3$, and consider

$$
\begin{aligned}
& M_{5}=\{(8 t+2,4 t+2),(8 t+1,4 t+3), \ldots,(6 t+3,6 t+1)\}, \\
& M_{6}=\{(6 t+2,2 t+1),(2 t, 1),(3 t+1,3 t)\}, \\
& M_{7}=\{(4 t+1,2),(4 t, 3), \ldots,(3 t+2, t+1)\}, \\
& M_{8}=\{(3 t-1, t+2),(3 t-2, t+3), \ldots,(2 t+2,2 t-1)\} .
\end{aligned}
$$

Note that each one of these sets is a matching of $K_{n}$. A simple inspection reveals that each $M_{i}$ is an embedded matching of $\left(K_{n}, \omega\right)$. We apply Lemma 3.2 again. Therefore, $M=\bigcup_{i=5}^{8} M_{i}$ is an embedded matching of $\left(K_{n}, \omega\right)$ with maximum cardinality, by Proposition 2.3 .

The proof of Theorem 3.3 is complete.

\section{From EMBEDDED MATCHING TO SHORT COVERING}

4.1. Outline of the construction. We begin this section with an outline of the proof of Theorem 1.1. Recall that the set of the square numbers

$$
\mathbb{F}_{q}^{\square}=\left\{\gamma^{2} \in \mathbb{F}_{q}^{*}: \gamma \in \mathbb{F}_{q}^{*}\right\}
$$

is a subgroup of the multiplicative group $\mathbb{F}_{q}^{*}$. The method also uses the following subset,

$$
\mathcal{E}=\left\{(1, y, z) \in \mathbb{F}_{q}^{3}: 1, y, z \text { are pairwise distinct, } y z \neq 0 \text { and } y, z \notin \mathbb{F}_{q}^{\square}\right\},
$$

which plays a central role in the proof.

The space $\mathbb{F}_{q}^{3}$ is naturally partitioned into two subsets: $\mathbb{F}_{q}^{*} \cdot \mathcal{E}$ and $\mathbb{F}_{q}^{3} \backslash\left(\mathbb{F}_{q}^{*} \cdot \mathcal{E}\right)$. Suppose that there is a way of finding short covering of $\mathcal{E}$ (a short covering of $\mathbb{F}_{q}^{*} \cdot \mathcal{E}$, 
equivalently) and a short covering of $\mathbb{F}_{q}^{3} \backslash\left(\mathbb{F}_{q}^{*} \cdot \mathcal{E}\right)$. Thus we get a short covering of the whole space $\mathbb{F}_{q}^{3}$. How do we find a systematical way for an arbitrary $q$ ? Roughly speaking, the answer is given by a construction based on two main steps, namely,

Step 1: Take $m=(q-1) / 2$ and pick a maximum embedded matching of $\left(K_{m-1}, \omega\right)$. Then a set $\mathcal{X}$ is generated from this matching by Theorem 4.4 below. The set $\mathcal{X}$ will be a short covering of "most" vectors in $\mathcal{E}$.

Step 2: Build a set $\mathcal{Z}$ that contains $\mathcal{X}$ and also satisfies additional conditions given by Theorem 4.5. We will see that

$$
\{(1,1,0),(1,0,1),(0,1,1)\} \cup \mathcal{Z}
$$

is a short covering of $\mathbb{F}_{q}^{3} \backslash\left(\mathbb{F}_{q}^{*} \cdot \mathcal{E}\right)$.

4.2. The connections. Given two vectors $x=\left(x_{1}, x_{2}, x_{3}\right)$ and $y=\left(y_{1}, y_{2}, y_{3}\right)$ in $\mathbb{F}_{q}^{3}$, the Hamming distance between the words $x$ and $y$ is

$$
d(x, y)=\left|\left\{i: x_{i} \neq y_{i}\right\}\right| .
$$

Remark 4.1. This distance allows us to reformulate the short covering defined in (1.1) as follows: a subset $\mathcal{H}$ in $\mathbb{F}_{q}^{3}$ is a short covering of $\mathbb{F}_{q}^{3}$ if and only if

$$
\bigcup_{h \in \mathcal{H}} \bigcup_{\alpha \in \mathbb{F}_{q}}\left\{x \in \mathbb{F}_{q}^{3}: d(x, \alpha h) \leq 1\right\}=\mathbb{F}_{q}^{3} \text {. }
$$

The four projections

$$
(x, y, z) \rightarrow x,(x, y, z) \rightarrow y,(x, y, z) \rightarrow z, \text { and }(x, y, z) \rightarrow(y, z)
$$

are denoted by $\pi_{1}, \pi_{2}, \pi_{3}$ and $\pi$, respectively.

The concept of linearly independent vectors will be used as a "bridge" linking embedded matching and short covering. The next result is concerned on the covering of $\mathbb{F}_{q}^{*} \cdot \mathcal{E}$. Note that a set $\mathcal{X}$ is a short covering of $\mathbb{F}_{q}^{*} \cdot \mathcal{E}$ if only if $\mathcal{X}$ is a short covering of $\mathcal{E}$.

Theorem 4.2. If $\mathcal{X}$ is a subset of $\mathbb{F}_{q}^{3}$ such that:

(1) $\pi_{1}(\mathcal{X})=\{1\}$

(2) $\pi_{2}(u) \neq \pi_{3}(u)$, for every $u \in \mathcal{X}$,

(3) $\pi(\mathcal{X}) \subset\left(\mathbb{F}_{q}^{\square} \backslash\{1\}\right) \times\left(\mathbb{F}_{q}^{\square} \backslash\{1\}\right)$,

(4) the vectors in $\pi(\mathcal{X})$ are pairwise linearly independent.

Then $\mathcal{X}$ is a short covering of exactly $|\mathcal{X}| \cdot(q-1) / 2$ vectors of $\mathcal{E}$.

Proof. Let $t=(q-1) / 2$, and consider $\mathbb{F}_{q}^{*} \backslash \mathbb{F}_{q}^{\square}=\left\{r_{1}, \ldots, r_{t}\right\}$.

Fact 1 . Every vector $(1, \beta, \gamma)$ in $\mathcal{X}$ is a short covering of $t$ vectors of $\mathcal{E}$, namely, $\left(1, r_{1} \beta, r_{1} \gamma\right), \ldots,\left(1, r_{t} \beta, r_{t} \gamma\right)$. Indeed, since $\mathbb{F}_{q}^{\square}$ is a subgroup of $\mathbb{F}_{q}^{*}$, both numbers $\alpha \beta$ and $\alpha \gamma$ belong to $\mathbb{F}_{q}^{\square}$ for every $\alpha \in \mathbb{F}_{q}^{\square}$, but $r_{i} \beta$ and $r_{i} \gamma$ do not belong to $\mathbb{F}_{q}^{\square}$, and $r_{i} \beta \neq r_{i} \gamma$ for every $i, 1 \leq i \leq t$. Thus the distinct vectors $\left(1, r_{1} \beta, r_{1} \gamma\right), \ldots,\left(1, r_{t} \beta, r_{t} \gamma\right)$ are all the vectors of $\mathcal{E}$ covered by $(1, \beta, \gamma)$.

Fact 2. A vector of $\mathcal{E}$ cannot be simultaneously covered (shortly) by two distinct vectors of $\mathcal{X}$. Indeed, suppose that $(1, y, z) \in \mathcal{E}$ is covered by the vectors $(1, \beta, \gamma)$ and $\left(1, \beta^{\prime}, \gamma^{\prime}\right)$ of $\mathcal{X}$. By Fact 1 , there would be $r, r^{\prime} \in \mathbb{F}_{q}^{*} \backslash \mathbb{F}_{q}^{\square}$ such that $y=r \beta=r^{\prime} \beta^{\prime}$ and $z=r \gamma=r^{\prime} \gamma^{\prime}$. However, $(\beta, \gamma)=\left(r^{-1} r^{\prime}\right) \cdot\left(\beta^{\prime}, \gamma^{\prime}\right)$, which is a contradiction with the fourth hypothesis. 
Remark 4.3. Theorem 4.2 gives us the following characterization. Let $\mathcal{X}$ be a set which satisfies the hypothesis of Theorem 4.2 and $u \in \mathcal{X}$. Thus a vector $v \in \mathcal{E}$ is covered (shortly) by $u$ if and only if there is $r \in \mathbb{F}_{q}^{*} \backslash \mathbb{F}_{q}^{\square}$ such that $v$ and $r \cdot u$ coincide in both the second and third coordinates.

A connection between embedded matchings and short coverings is described as follows. This link will allow us to get a new upper bound for covering codes.

Theorem 4.4. Let $q=2 m+1$ be an odd prime power, and let $\xi$ be a generator of $\mathbb{F}_{q}^{*}$. If $M=\left\{\left(a_{1}, b_{1}\right), \ldots,\left(a_{s}, b_{s}\right)\right\}$ is an embedded matching of $\left(K_{m-1}, \omega\right)$, then the set

$$
\mathcal{X}=\bigcup_{i=1}^{s}\left\{\left(1, \xi^{2 a_{i}}, \xi^{2 b_{i}}\right),\left(1, \xi^{2 b_{i}}, \xi^{2 a_{i}}\right)\right\}
$$

is a short covering of $(q-1)$ s vectors in $\mathcal{E}$.

Proof. The result is an application of Theorem 4.2. Indeed, the first hypothesis holds trivially. Since $M$ is a matching of $K_{m-1}$, the second hypothesis is satisfied too. Take

$$
\mathcal{Y}=\pi(\mathcal{X})=\bigcup_{i=1}^{s}\left\{\left(\xi^{2 a_{i}}, \xi^{2 b_{i}}\right),\left(\xi^{2 b_{i}}, \xi^{2 a_{i}}\right)\right\}
$$

Note that the projections $\pi_{2}(\mathcal{X})$ and $\pi_{3}(\mathcal{X})$ are subsets of $\mathbb{F}_{q}^{\square} \backslash\{1\}$, because each one of their elements is written as $\xi^{\alpha}$ for some even number $\alpha, 2 \leq \alpha \leq q-3$, thus $\mathcal{Y} \subset\left(\mathbb{F}_{q}^{\square} \backslash\{1\}\right) \times\left(\mathbb{F}_{q}^{\square} \backslash\{1\}\right)$.

Fact 1. Let $(x, y)$ and $(z, w)$ be vectors in $\mathbb{F}_{q}^{*} \times \mathbb{F}_{q}^{*}$, and write $(x, y)=\left(\xi^{b}, \xi^{c}\right)$ and $(z, w)=\left(\xi^{d}, \xi^{e}\right)$, where $\xi$ is a generator of $\mathbb{F}_{q}^{*}$ and $b, c, d, e \in[q-1]$. Then $(x, y)$ and $(z, w)$ are linearly dependent if and only if $b-c=d-e$.

Indeed, since the groups $\left(\mathbb{F}_{q}^{*}, \cdot\right)$ and $\left(\mathbb{Z}_{q-1},+\right)$ are isomorphic, the vectors $(x, y)$ and $(z, w)$ are linearly dependent if and only if there is $a \in[q-1]$ such that $\xi^{a}\left(\xi^{b}, \xi^{c}\right)=\left(\xi^{d}, \xi^{e}\right)$, or equivalently, $a+b=d$ and $a+c=e$. Hence Fact 1 follows.

It remains to prove that the vectors in $\mathcal{Y}$ are pairwise linearly independent in $\left(\mathbb{F}_{q}^{\square} \backslash\{1\}\right) \times\left(\mathbb{F}_{q}^{\square} \backslash\{1\}\right)$. In view of Fact 1 and the shape of the elements in $\mathcal{Y}$, it is sufficient to show that:

(i) for every $j \neq i, a_{i}-b_{i} \neq a_{j}-b_{j}$,

(ii) for each $j \neq i, b_{i}-a_{i} \neq b_{j}-a_{j}$,

(iii) $a_{i}-b_{i} \neq b_{j}-a_{j}$, for all $i, j, 1 \leq i \leq j \leq s$.

This is the motivation for Definition 2.1 Since $M$ is an embedded matching in $\left(K_{m-1}, \omega\right)$, we have that $a_{i}-b_{i}=\omega\left(a_{i}, b_{i}\right) \neq \omega\left(a_{j}, b_{j}\right)=a_{j}-b_{j}$ and (i) is proved. The item (ii) is derived from (i) immediately. Item (iii) is trivial. By Theorem 4.2 . the statement follows.

Theorem 4.5. Let $\mathcal{Z}$ be a subset of $\mathbb{F}_{q}^{3}$ which satisfies the following properties:

(1) $\pi_{1}(\mathcal{Z})=\{1\}$,

(2) $\mathbb{F}_{q}^{\square} \backslash\{1\} \subset \pi_{2}(\mathcal{Z})$,

(3) $\mathbb{F}_{q}^{\square} \backslash\{1\} \subset \pi_{3}(\mathcal{Z})$.

Then the set $\{(1,1,0),(1,0,1),(0,1,1)\} \cup \mathcal{Z}$ is a short covering of $\mathbb{F}_{q}^{3} \backslash\left(\mathbb{F}_{q}^{*} \cdot \mathcal{E}\right)$.

Proof. Choose an arbitrary vector $u$ in $\mathbb{F}_{q}^{3} \backslash\left(\mathbb{F}_{q}^{*} \cdot \mathcal{E}\right)$. We analyze three cases. 
Case 1. The vector $u$ has at least two coincident coordinates. In this case, there are $\alpha$ and $\beta$ in $\mathbb{F}_{q}$ such that $u$ assumes one of the forms: $u_{1}=(\alpha, \alpha, \beta), u_{2}=(\alpha, \beta, \alpha)$ or $u_{3}=(\beta, \alpha, \alpha)$. Since

$$
d\left(u_{1}, \alpha \cdot(1,1,0)\right) \leq 1, d\left(u_{2}, \alpha \cdot(1,0,1)\right) \leq 1, d\left(u_{3}, \alpha \cdot(0,1,1)\right) \leq 1,
$$

$\{(1,1,0),(1,0,1),(0,1,1)\}$ is always a short covering of $u$, by Remark 4.1 .

Case 2. The vector $u$ has at least one of the coordinates as null. In this case, $u$ is written as: $u_{1}=(\alpha, \beta, 0), u_{2}=(\alpha, 0, \beta)$, or $u_{3}=(0, \alpha, \beta)$. By Remark 4.1, $u_{1}, u_{2}$, and $u_{3}$ are covered (shortly) by $\{(1,1,0),(1,0,1),(0,1,1)\}$, since

$$
d\left(u_{1}, \alpha \cdot(1,1,0)\right) \leq 1, d\left(u_{2}, \alpha \cdot(1,0,1)\right) \leq 1, d\left(u_{3}, \alpha \cdot(0,1,1)\right) \leq 1 .
$$

The impact of the set $\{(1,1,0),(1,0,1),(0,1,1)\}$ on short covering was established in 13 .

Case 3. Otherwise, $u=(\alpha, \beta, \gamma)$ is a vector in $\mathbb{F}_{q}^{3}$ such that $\alpha, \beta, \gamma$ are pairwise distinct and non-null. Note that $u$ is covered (shortly) by a vector $v$ if and only if $\left(1, \alpha^{-1} \beta, \alpha^{-1} \gamma\right)$ is covered by $v$ also. Therefore, we can assume that $u=(1, \beta, \gamma)$, with $1, \beta$ and $\gamma$ pairwise distinct, $\beta, \gamma \neq 0$ and $\beta \in \mathbb{F}_{q}^{\square} \backslash\{1\}$ or $\gamma \in \mathbb{F}_{q}^{\square} \backslash\{1\}$. If $\beta \in \mathbb{F}_{q}^{\square}$, by hypothesis, there is a vector of type $v=(1, \beta, \cdot) \in \mathcal{Z}$ such that $d(u, v) \leq 1$. Since the case where $\gamma \in \mathbb{F}_{q}^{\square}$ is similar, the proof is complete.

\section{UPPER BOUND FOR SHORT COVERINGS}

Lemma 5.1. If $q \equiv 3(\bmod 4)$ is a prime power, then

$$
c(q) \leq(q+3) / 2 .
$$

Proof. Consider

$$
\mathcal{X}=\left\{(1, y, z) \in \mathbb{F}_{q}^{3}: y, z \in \mathbb{F}_{q}^{\square} \backslash\{1\} \text { and } z=y^{-1}\right\} .
$$

We claim that

$$
\mathcal{H}_{1}=\{(1,1,0),(1,0,1),(0,1,1)\} \cup \mathcal{X}
$$

is a short covering of $\mathbb{F}_{q}^{3}$.

Step 1. The set $\mathcal{H}_{1}$ is a short covering of $\mathcal{E}$. Note that for each $(1, y, z) \in \mathcal{X}$, $y \neq z$ holds. Suppose for a contradiction that $y=z=y^{-1}$, thus $y^{2}=1$. Since the environment is a field, the only solutions are $y= \pm 1$. By construction $y \neq 1$, which implies that $y=-1$. This is a contradiction with the well-known statement: -1 is a square number in $\mathbb{F}_{q}$ if and only if $q \equiv 1(\bmod 4)($ see $[6])$.

The vectors in $\pi(\mathcal{X})$ are pairwise linearly independent. Indeed, if there are two distinct vectors $(y, z)$ and $\left(y^{\prime}, z^{\prime}\right) \in \pi(\mathcal{X})$ such that $(y, z)=\alpha \cdot\left(y^{\prime}, z^{\prime}\right)$ for any $\alpha \in \mathbb{F}_{q}^{\square}$, then $\alpha= \pm 1$, a contradiction. The set $\mathcal{X}$ satisfies Theorem 4.2. Since $|\mathcal{X}|=(q-3) / 2$, the set $\mathcal{X}$ is a short covering of $\mathcal{E}$.

Step 2. By construction of $\mathcal{X}$, it follows that $\pi_{2}(\mathcal{X})=\pi_{3}(\mathcal{X})=\mathbb{F}_{q}^{\square} \backslash\{1\}$. Theorem 4.5 implies that $\mathcal{H}_{1}$ is a short covering of $\mathbb{F}_{q}^{3} \backslash\left(\mathbb{F}_{q}^{*} \cdot \mathcal{E}\right)$ also.

Example 5.2. Let us build a short covering of $\mathbb{Z}_{11}^{3}$ by using Lemma 5.1 The set $\mathcal{X}=\{(1,3,4),(1,4,3),(1,5,9),(1,9,5)\}$ given by (5.1) satisfies Theorem 4.2, then all 20 vectors of the corresponding $\mathcal{E}$ are covered by $\mathcal{X}$. Since $\pi(\mathcal{X})=\left(\mathbb{Z}_{11}^{\square} \backslash\{1\}\right) \times$ $\left(\mathbb{Z}_{11}^{\square} \backslash\{1\}\right)$, the set $\mathcal{X}$ also satisfies Theorem 4.5. Hence,

$$
\{(1,1,0),(1,0,1),(0,1,1),(1,3,4),(1,4,3),(1,5,9),(1,9,5)\}
$$


is a short covering of $\mathbb{Z}_{11}^{3}$ and then $c(11) \leq 7$.

The next constructions arise from suitable embedded matchings.

Lemma 5.3. Let $q \equiv 3(\bmod 4)$ be a prime power such that $q=2 m+1$ with $m \equiv 1(\bmod 8)$ or $m \equiv 3(\bmod 8)$. Then

$$
c(q) \leq(q+3) / 2 .
$$

Proof. Choose $\left\{\left(a_{1}, b_{1}\right), \ldots,\left(a_{s}, b_{s}\right)\right\}$ an optimal embedded matching of $\left(K_{m-1}, \omega\right)$. Let $\xi$ be a generator of $\mathbb{F}_{q}^{*}$ and consider the set $\mathcal{X}$ given in (4.2). Let us show that

$$
\mathcal{H}_{2}=\{(1,1,0),(1,0,1),(0,1,1)\} \cup \mathcal{X}
$$

is a short covering of $\mathbb{F}_{q}^{3}$.

Step 1 . The set $\mathcal{H}_{2}$ is a short covering of $\mathcal{E}$. Indeed, by Theorem 3.3 it follows that $s=(q-3) / 4$. Theorem 4.4 implies that $\mathcal{X}$ is a short covering of exactly $[(q-1) \cdot(q-3)] / 4=|\mathcal{E}|$ vectors of $\mathcal{E}$.

Step 2. The set $\mathcal{H}_{2}$ is a short covering of $\mathbb{F}_{q}^{3} \backslash\left(\mathbb{F}_{q}^{*} \cdot \mathcal{E}\right)$. Indeed, by the construction of $\mathcal{X}$, the projection $\pi_{i}(\mathcal{X})$ is contained in $\mathbb{F}_{q}^{\square} \backslash\{1\}$ and $\left|\pi_{i}(\mathcal{X})\right|=(q-3) / 2=$ $\left|\mathbb{F}_{q}^{\square} \backslash\{1\}\right|$. Hence, $\pi_{i}(\mathcal{X})=\mathbb{F}_{q}^{\square} \backslash\{1\}$ for $i=2$ and $i=3$. Finally, Theorem 4.5 implies that $\mathcal{H}_{2}$ is a short covering of $\mathbb{F}_{q}^{3} \backslash\left(\mathbb{F}_{q}^{*} \cdot \mathcal{E}\right)$.

Example 5.4. Take the finite field $\mathbb{Z}_{19}$. According to Theorem 3.3, there is an optimal embedded matching of $\left(K_{8}, \omega\right)$ with four edges. For instance, choose $\{(2,1),(7,5),(6,3),(8,4)\}$. By using 2 as a generator of $\mathbb{Z}_{19}^{*}$, Theorem 4.4 produces

$$
\mathcal{X}=\{(1,16,4),(1,4,16),(1,6,17),(1,17,6),(1,11,7),(1,7,11),(1,5,9),(1,9,5)\},
$$

which is a short covering of exactly $4 \cdot 18=72$ elements of the corresponding $\mathcal{E}$. Since $|\mathcal{E}|=72$, the set $\mathcal{E}$ is totally covered by $\mathcal{X}$.

Since $\mathbb{Z}_{19}^{\square}=\{1,4,5,6,7,9,11,16,17\}$, the condition $\pi_{i}(\mathcal{X})=\mathbb{Z}_{19}^{\square} \backslash\{1\}$ holds for $i=2$ or $i=3$. By applying Theorem 4.5. the set $\{(1,1,0),(1,0,1),(0,1,1)\} \cup \mathcal{X}$ covers $\mathbb{Z}_{19}^{3} \backslash\left(\mathbb{Z}_{19}^{*} \cdot \mathcal{E}\right)$. The desired covering is obtained.

It is worth mentioning that a sharp construction for $c(7)$ can be derived from the previous result, as observed in [10].

Part of Lemma 5.1 is reproved in Lemma 5.3 by using embedded matchings. For the remaining part, where $m \equiv 5$ or $7(\bmod 8)$, there is no hope of applying this approach because Theorem 3.3 gives us only $\Omega(m-1)=(m-1) / 2-1$ in these cases. The last case depends on embedded matching.

Lemma 5.5. Let $q$ be a prime power such that $q \equiv 1(\bmod 4)$, then

$$
c(q) \leq(q+5) / 2 .
$$

Proof. Write $q=2 m+1$ for some even number $m$. Pick an optimal embedded matching $M=\left\{\left(a_{1}, b_{1}\right), \ldots,\left(a_{s}, b_{s}\right)\right\}$ of $\left(K_{m-1}, \omega\right)$. Since $m-1$ is odd, there is an element in $[m-1]$, say $k$, which is not matched by $M$. The construction of a short covering is divided into two steps, as mentioned in Subsection 4.1 .

Step 1. Let $\xi$ be a generator of $\mathbb{F}_{q}^{*}$, and consider the set $\mathcal{X}$ defined in (4.2). The set

$$
\mathcal{Z}=\mathcal{X} \cup\left\{\left(1, \xi^{2 k},-\xi^{2 k}\right),\left(1,-\xi^{2 k}, \xi^{2 k}\right)\right\}
$$


is a short covering of $\mathcal{E}$. Indeed, Theorem 3.3 implies that

$$
s=\Omega(m-1)=\lfloor(m-1) / 2\rfloor=(q-5) / 4 .
$$

By Theorem 4.4 the set $\mathcal{X}$ is a short covering of $[(q-5)(q-1)] / 4$ vectors of $\mathcal{E}$. Since $|\mathcal{E}|=[(q-3)(q-1)] / 4$, we find an obstacle: how to cover the $(q-1) / 2$ remaining vectors in $\mathcal{E}$. The characterization of these vectors is described below.

Fact 1. Each vector of $\mathcal{E}$ not covered by $\mathcal{X}$ must have the form $(1, y,-y)$, for some $y \in \mathbb{F}_{q}^{*} \backslash \mathbb{F}_{q}^{\square}$. Suppose for a contradiction that $(1, y,-y) \in \mathcal{E}$ is covered by $\left(1, \xi^{a}, \xi^{b}\right) \in \mathcal{X}$. By the characterization obtained in Theorem 4.2, there exists $r \in$ $\mathbb{F}_{q}^{*} \backslash \mathbb{F}_{q}^{\square}$ such that $(y,-y)=r \cdot\left(\xi^{a}, \xi^{b}\right)$. Hence $\left(\xi^{a}, \xi^{b}\right)=-1 \cdot\left(\xi^{b}, \xi^{a}\right)$, a contradiction with the fact that the vectors in $\pi(\mathcal{X})$ are pairwise linearly independent.

Fact 2. The vector $\left(1, \xi^{2 k},-\xi^{2 k}\right)$ is a short covering of each vector in $\mathcal{E}$ of type $(1, y,-y)$. Recall that $\mathbb{F}_{q}^{*} \backslash \mathbb{F}_{q}^{\square}=\left\{r_{1}, \ldots, r_{t}\right\}$, then the bijection $\phi\left(r_{i}\right)=r_{i} \xi^{2 k}$ from $\mathbb{F}_{q}^{*} \backslash \mathbb{F}_{q}^{\square}$ to $\mathbb{F}_{q}^{*} \backslash \mathbb{F}_{q}^{\square}$ implies that $y=r_{j} \xi^{2 k}$ for some $j, 1 \leq j \leq t$. The vectors $(1, y,-y)$ and $r_{j}\left(1, \xi^{2 k},-\xi^{2 k}\right)$ differ just in the first coordinate.

Step 2. The set

$$
\mathcal{H}_{3}=\{(1,1,0),(1,0,1),(0,1,1)\} \cup \mathcal{Z}
$$

is a short covering of $\mathbb{F}_{q}^{3} \backslash\left(\mathbb{F}_{q}^{*} \cdot \mathcal{E}\right)$. We have already showed that $\pi_{i}(\mathcal{X})$ is a subset of $\mathbb{F}_{q}^{\square} \backslash\{1\}$, for $i=2$ or $i=3$. Now, the elements of $\pi_{i}(\mathcal{X})$ are pairwise distinct and $\xi^{2 k} \notin \pi_{i}(\mathcal{X})$, for $i=2$ or $i=3$; but $\left|\pi_{i}(\mathcal{Z})\right|=(q-3) / 2$, that is, $\pi_{i}(\mathcal{Z})=\mathbb{F}_{q}^{\square} \backslash\{1\}$. Theorem 4.5 implies that $\mathcal{H}_{3}$ is also a short covering of $\mathbb{F}_{q}^{3} \backslash\left(\mathbb{F}_{q}^{*} \cdot \mathcal{E}\right)$. Therefore $\mathcal{H}_{3}$ is a short covering of $\mathbb{F}_{q}^{3}$.

Remark 5.6. In Lemma 5.5, we proved that $\mathcal{X} \cup\left\{\left(1, \xi^{2 k},-\xi^{2 k}\right)\right\}$ is a short covering of $\mathcal{E}$. In order to build a short covering of $\mathbb{F}_{q}^{3} \backslash\left(\mathbb{F}_{q}^{*} \cdot \mathcal{E}\right)$, the vector $\left(1,-\xi^{2 k}, \xi^{2 k}\right)$ is indispensable, because $\mathcal{H}^{\prime}=\{(1,1,0),(1,0,1),(0,1,1)\} \cup \mathcal{X} \cup\left\{\left(1, \xi^{2 k},-\xi^{2 k}\right)\right\}$ is not a short covering of $u=\left(1, y, \xi^{2 k}\right)$ for some $y \in \mathbb{F}_{q}^{*} \backslash \mathbb{F}_{q}^{\square}$. Indeed, suppose for a contradiction that there is a vector $v \in \mathcal{H}^{\prime}$ that is a short covering of $u$. Clearly, $v \notin\{(1,1,0),(1,0,1),(0,1,1)\}$. So $v=(1, b, c)$ with $b, c \in \mathbb{F}_{q}^{\square}$ and there is a scalar $\alpha \in \mathbb{F}_{q}^{*}$ such that $d(u, \alpha v) \leq 1$. If $\alpha \in \mathbb{F}_{q}^{\square}$, then $\alpha v$ has the three coordinates in $\mathbb{F}_{q}^{\square}$. Since $y \in \mathbb{F}_{q}^{*} \backslash \mathbb{F}_{q}^{\square}$, we have $\alpha=1$ and $\alpha c=\xi^{2 k}$, so $c=\xi^{2 k}$, a contradiction. Otherwise, if $\alpha \in \mathbb{F}_{q}^{*} \backslash \mathbb{F}_{q}^{\square}$, then $\alpha v$ has the three coordinates in $\mathbb{F}_{q}^{*} \backslash \mathbb{F}_{q}^{\square}$. Since $1, \xi^{2 k} \in \mathbb{F}_{q}^{\square}$ we have $d(u, \alpha v) \geq 2$, another contradiction.

Example 5.7. The short covering for $\mathbb{F}_{13}^{3}$ illustrates the above steps. Since $13=$ $2 \cdot 6+1$, we take $M=\{(2,1),(5,3)\}$ an optimal embedded matching of $\left(K_{5}, \omega\right)$. The number 2 is a generator of $\mathbb{Z}_{13}^{*}$. By Theorem 4.4, the set

$$
\begin{aligned}
\mathcal{X} & =\left\{\left(1,2^{2 \cdot 2}, 2^{2 \cdot 1}\right),\left(1,2^{2 \cdot 1}, 2^{2 \cdot 2}\right),\left(1,2^{2 \cdot 5}, 2^{2 \cdot 3}\right),\left(1,2^{2 \cdot 3}, 2^{2 \cdot 5}\right)\right\} \\
& =\{(1,3,4),(1,4,3),(1,10,12),(1,12,10)\}
\end{aligned}
$$

is a short covering of $2 \cdot 12=24$ elements of $\mathcal{E}$. Since $|\mathcal{E}|=30$, we need to cover 6 remaining vectors of $\mathcal{E}$. By Fact 1 of Lemma 5.5, these six vectors are: $(1,2,11)$, $(1,5,8),(1,6,7),(1,7,6),(1,8,5),(1,11,2)$. They are covered by $\left(1,2^{2 \cdot 4},-2^{2 \cdot 4}\right)=$ $(1,9,4)$. Note that we have to choose $\left(1,2^{2 \cdot 4},-2^{2 \cdot 4}\right)$ because 4 is the only element of [5] which was not matched by $M$.

By step 1 of Lemma 5.5, the set $\mathcal{X} \cup\{(1,9,4)\}$ is a short covering of $\mathcal{E}$. It remains to show how to cover $\mathbb{Z}_{13}^{3} \backslash\left(\mathbb{Z}_{13}^{*} \cdot \mathcal{E}\right)$. For this purpose, we include the vectors $(1,9,4)$ 
and $(1,4,9)$ in $\mathcal{X}$, then

$$
\mathcal{Z}=\{(1,3,4),(1,4,3),(1,10,12),(1,12,10),(1,9,4),(1,4,9)\} .
$$

Because $\mathcal{Z}$ satisfies Theorem 4.5. $\{(1,1,0),(1,0,1),(0,1,1)\} \cup \mathcal{Z}$ is a short covering of $\mathbb{Z}_{13}^{3} \backslash\left(\mathbb{Z}_{13}^{*} \cdot \mathcal{E}\right)$.

Thus,

$$
\{(1,1,0),(1,0,1),(0,1,1),(1,3,4),(1,4,3),(1,10,12),(1,12,10),(1,9,4),(1,4,9)\}
$$

is a short covering of $\mathbb{Z}_{13}^{3}$, and $c(13) \leq 9$ holds.

Proof of Theorem 1.1. The proof is an immediate consequence of Lemmas [5.1] and 5.5 .

\section{REFERENCES}

1. S. Banerjee, A. Datta Chowdhury, and S.K. Ghosh, Efficient algorithms for variants of weighted matching and assignment problems, Math. Comput. Sci., vol. 1 (2008), 673-688. MR2415165 (2009i:68102)

2. W.A. Carnielli, On covering and coloring problems for rook domains, Discrete Math., vol. 57 (1985), 9-16. MR816044 (86m:05033)

3. G. Cohen, I. Honkala, S. Litsyn, and A. Lobstein, Covering Codes, North-Holland, Amsterdam, (1997). MR1453577(99b:94059)

4. T.J. Dickson, A covering problem concerning abelian groups, J. London Math. Soc., vol. 3 (1971), 222-232. MR0272889 (42:7770)

5. M. Garey and D. Johnson, Computers and intractability: a guide to the theory of NPcompleteness, W.H. Freeman and Company, (1979). MR519066 (80g:68056)

6. I.N. Herstein, Topics in Algebra, J. Wiley \& Sons, Singapore, (1993).

7. J.G. Kalbfleisch and R.G. Stanton, A Combinatorial Problem in Matching, J. London Math. Soc., vol. 44 (1969), 60-64. MR0231734(38:62)

8. G. Kéri, Tables for bound on covering codes, homepage: http://www.sztaki.hu/keri/accessed (2011).

9. L. Lovász and M.D. Plummer, Matching theory, AMS Chelsea Publishing, Providence, (2009). MR2536865

10. A.N. Martinhão and E.L. Monte Carmelo, A new exact value on short covering, manuscript in preparation, (2011).

11. C. Mendes, E.L. Monte Carmelo, and M.V. Poggi de Aragão, Bounds for short covering codes and reactive tabu search, Discrete Appl. Math. vol. 158 (2010), 522-533. MR2592458 (2011f:94164)

12. E.L. Monte Carmelo and C.F.X. De Mendonça Neto, Extremal problems on sum-free sets and coverings in tridimensional spaces, Aequationes Mathematicae, vol. 78 (2009), 101-112. MR2552526 (2010i:11100)

13. E.L. Monte Carmelo and I.N. Nakaoka, Short coverings in tridimensional spaces arising from sum-free sets, European J. Combin., vol. 29 (2008), 227-233. MR2368629 (2008j:11081)

14. I.N. Nakaoka and O.J.N.T.N. Santos, A covering problem over finite rings, Appl. Math. Letters, vol. 23 (2010), 322-326. MR2565199 (2011e:13049)

15. S.K. Zaremba, A covering theorem for abelian groups, J. London Math. Soc., vol. 26 (1951), 71-72. MR0038967(12:477a)

Departamento de Matemática, Universidade Estadual de Maringá, Brazil

E-mail address: and_nm@hotmail.com

Departamento de Matemática, Universidade Estadual de Maringá, Brazil

E-mail address: elmcarmelo@uem.br 